\title{
Expression of Odontoblastic-Related Genes in Human Dental Follicle Cells, Dental Pulp Stem Cells, and Oral Mucosal Cells
}

\author{
Makoto Takahashi, ${ }^{1}$ Yoichi Yamada, ${ }^{2}$ Ryotaro Ozawa, ${ }^{1}$ Morimichi Ohya, ${ }^{1}$ Kenji Ito, ${ }^{1}$ and \\ Minoru Ueda ${ }^{1}$
}

${ }^{1}$ Department of Oral and Maxillofacial Surgery, Nagoya University Graduate School of Medicine, Nagoya 466-8550, Japan

${ }^{2}$ Center for Genetic and Regenerative Medicine, National University Corporation Nagoya University School of Medicine, Nagoya 466-8550, Japan

Correspondence to :

Yoichi Yamada

E-mail : yyamada@ med.nagoya-u. ac.jp

Keywords :

dental follicle cells, dental pulp stem cells, odontoblast-related gene, real time RT-PCR, tissue engineering

\begin{abstract}
In the current studies, we examined the ability of human dental follicle cells (DFCs), dental pulp stem cells (DPSCs), and oral mucosal cells (OMCs) to form dentin by analyzing their expression of the odontoblast-specific genes, dentin sialophosphoprotein (DSPP) and dentin sialoprotein (DSP), and the mineralization-associated genes, alkaline phosphatase (ALP) and dentin matrix protein-1 (DMP-1). Phase contrast microscopy showed that DFCs, DPSCs, and OMCs exhibited spindle-shaped and fibroblastic morphologies. Also, more bone nodules were formed in DFCs than in DPSCs, whereas bone nodules were not found in OMCs. Analysis of gene expression by real-time reverse transcriptase-polymerase chain reaction showed a time-dependent increase in the expression of DSPP in differentiating DFCs and DPSCs, but almost no expression in OMCs. The expression of DSPP in DFCs was approximately 4 -fold higher than in DPSCs after 15 days in culture, and, almost 6 -fold higher after 27 days. In addition, the expression of DSP, DMP-1 and ALP was observed in both DFCs and DPSCs, with a slow increase until 15 days of differentiation, and, after 21 days, there was a rapid increase in the expression of these genes in DFCs. In contrast, expression of these genes was almost undetectable in OMCs and in undifferentiated DFCs, DPSCs and OMCs. These results suggest that, compared to DPSCs, DFCs have a superior capacity for dentin formation and mineralization and that they may be useful for the regeneration of dentin or tooth tissues using tissue engineering technology.
\end{abstract}

\section{Introduction}

The emerging fields of tissue engineering and regenerative medicine focus on the replacement or repair of lost or damaged tissues (1). This tissue engineering and regeneration requires three key ingredients: morphogenetic signals, including growth and differentiation factors; responding stem cells; and a scaffold of extracellular matrix (1). We have previously studied the use of mesenchymal stem cells along with platelet-rich plasma for the regeneration of bone and mucosal tissue for dermis regenera- tion $(2-8)$. We try to extend these studies into the area of dental tissue regeneration.

After physiologic stimulation or injury, such as caries and operative procedures, pulp stem cells may be mobilized to proliferate and differentiate into odontoblasts by morphogens secreted from surrounding dentin matrix. The damaged odontoblasts can be replaced by newly generated populations of odontoblasts derived from residual cycling stem cells in the pulp (9). Also, the dental pulp tissue contains some stem cells that retain the potential to replicate and 
differentiate into dentin-forming odontoblasts (10). In addition, during minor insults, odontoblasts differentiate into secondery dentin.

Dental follicle cells (DFCs) are one type of stem cells present in oral tissues that could be used for the egererating of dental tissues. The dental follicle is a loose connective tissue sac that surrounds the unerupted tooth. The DFCs originate from ectomesenchymal cells, and they contain subpopulations of mesenchymal progenitors that give rise to periodontal tissue, including cementum, periodontal ligament, and alveolar bone. However, few studies have characterized the roles of progenitor cells derived from DFCs. Wise et al. reported that DFCs express many genes related to tooth eruption (11) and DFCs have been reported to have the ability to differentiate into cementoblasts (12), but whether they have the capacity to differentiate into dentin is unknown.

In addition to DFCs, oral tissues also contain a population of putative postnatal stem cells in the dental pulp termed dental pulp stem cells (DPSCs) (10). The DPSCs are clonogenic and highly proliferative cells that have an ability to regenerate a dentin -pulp like-complex found in normal human teeth, including a mineralized matrix containing odontoblast-lined tubules and fibrous tissue containing blood vessels. DPSCs also express several bone markers in a manner similar to bone marrow stem cells, which are known for their high proliferative capacity and ability to differentiate into multiple cell types (10) as well as their stem cell-like qualities, including the capacity for self-renewal and multi-linkage differentiation.

In the current studies, we examined whether DFCs and DPSCs can differentiate into odontoblasts by following the expression of specific genetic markers. Specifically, we investigated the expression of odontoblast-specific genes, including dentin sialophosphoprotein (DSPP) and dentin sialoprotein (DSP), as well as genes expressed in mineralized tissue, including dentin matrix protein 1 (DMP-1) and alkaline phosphatase (ALP) (13).

The DSPP gene is found only in dentin and is a marker of odontoblast differentiation. DSP gene is transported via odontoblastic processes to the mineralization front shortly after synthesis. This gene is also a developmentally regulated protein that appears in terminally differentiated odontoblasts prior to the onset of mineralization (14).

ALP and DMP-1 are found not only in dentin but also in mineralized tissue. ALP is expressed constitutively by bone-forming cells and some periodontal ligament cells, and it is used as enzymatic marker of bone differentiation (15). DMP-1 was first cloned from the mineralized dentin matrix (16). Although the precise function of DMP-1 is has not been determined, in situ hybridization experiments have shown that it is expressed in both osteoblasts and odontoblasts (17).

To study the expression of differentiation of DFCs and DPSCs, we used real-time reverse transcriptase -polymerase chain reaction (RT-PCR) (18) to analyze the expression of these four marker genes. RT -PCR allows accurate and reproducible determination of the gene copy number, and, unlike standard $\mathrm{PCR}$, this method does not require post-PCR sample handling, preventing product carry-over contamination and increasing assay throughput (19). Our results show that DFCs and DPSCs might have the ability to form dentin and, therefore, may be useful for the regeneration of dentin or tooth tissues using tissue engineering technologies.

\section{Materials and Methods}

\section{Cell culture and RNA collection}

Normal human impacted third molars were collected from adults (13 to 15 years of age) at Nagoya University Hospital. All materials were used with the patients' informed consent. Dental follicle and pulp were separated by visual inspection immediately after extraction (Fig. 1). The isolated tissues were placed in $75 \mathrm{~cm}^{2}$ flasks and cultured for 20 to 30 days in conditioning medium (CM; Dulbecco's modified Eagle medium supplemented with $10 \%$ fetal bovine serum, $2 \mathrm{mM}$ glutamine, and 0.05 units $/ \mathrm{ml}$ penicillin) at $37^{\circ} \mathrm{C}$ in a humidified atmosphere containing $5 \%$ $\mathrm{CO}_{2}$.

Cells obtained from the primary culture were 


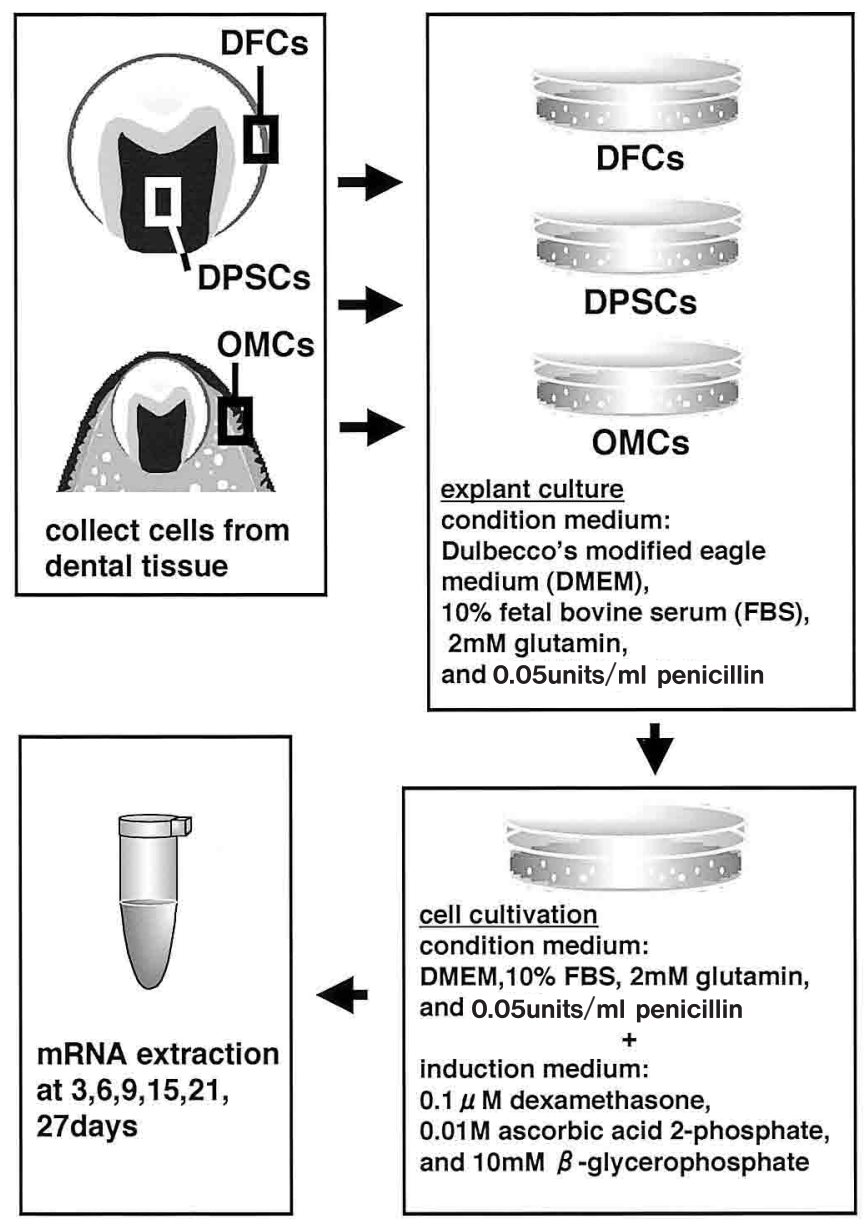

Fig. 1. Schema of experimental design. plated in a $100 \mathrm{~cm}^{2}$ tissue culture plate at a density of $3.1 \times 10^{3}$ cells $/ \mathrm{cm}^{2}\left(2.43 \times 10^{5}\right.$ cells per dish). To induce these cells to form hard tissue, the medium was changed to induction medium (IM ; CM supplemented with $0.1 \mu \mathrm{M}$ dexamethasone, $10 \mathrm{mM} \beta$-glycerophospate, and $0.05 \mathrm{M}$ ascorbic acid-2-phosphate). After 3, $6,9,15,21$, and 27 days, cultures were rinsed with phosphate-buffered saline (PBS) and total RNA was extracted using an RNeasy Mini Kit (QIAGEN GmbH, Hilden, Germany).

\section{Analysis by real-time $R T-P C R$}

Real-time RT-PCR was performed using a 7000 sequence detector (Perkin-Elmer, Wellesley, MA, USA). The reaction mixture was prepared according to the manufacture's protocol to give a final concentration of $1 \mathrm{x}$ avian myeloblastosis virus. Each $50 \mu 1$ multiplex PCR reaction contained $200 \mathrm{ng}$ RNA, Tfl reaction buffer, $0.2 \mathrm{mM}$ dNTPs, $1.5 \mathrm{mM} \mathrm{MgSO}_{4}, 0.1$ unit/ml avian myeloblastosis virus reverse transcriptase, and 0.1 unit/ml Tfl DNA polymerase probe (PCR-Access, Promega, Tokyo, JPN) as described by Gibson et al. (19). DNA amplification was performed with the following thermal cycling profile : $50^{\circ} \mathrm{C}$ for $2 \mathrm{~min}, 60^{\circ} \mathrm{C}$ for $30 \mathrm{~min}, 95^{\circ} \mathrm{C}$ for $5 \mathrm{~min}$, then 50 cycles of $95^{\circ} \mathrm{C}$ for $20 \mathrm{sec}$, and final extraction at $60^{\circ} \mathrm{C}$

Table 1. Sequences of Oligonucleotide primers and probes

\begin{tabular}{llc}
\hline primer of probe & \multicolumn{1}{c}{ sequence } & position \\
\hline \hline DSPP 163F & GCCATTCCAGTTCCTCAAGC & $163-187$ \\
DSPP 307R & CATGCACCAGGACACCACTT & $307-288$ \\
DSPP probe & TGATGGTTCCACTGGCATTTAACTCATCC & $225-283$ \\
DSP 3083F & GTGAAATATCTGGCAAACGAGACA & $3083-3106$ \\
DSP 3204R & CAGAGTTTCCAGTCCTGAGGTGTA & $3204-3181$ \\
DSP probe & ATTGCTGAACTTTGCGCCAATTCA & $3104-3127$ \\
hALP 783F & CCTCGTTGACACCTGGAAGAG & $783-803$ \\
hALP 822R & TTCCGTGCGGTTCCAGA & $822-806$ \\
hALP probe & TTCAAACCGAGATACAAGCACTCCCACTTC & $784-813$ \\
DMP1 6F & GATCAGCATCCTGCTCATGTTC & $6-27$ \\
DMP1 115R & GAGCCAAATGACCTTCCATT & $115-95$ \\
DMP1 probe & CCTGTGCTCTCCCAGTAACCAGGTATCAAA & $41-71$ \\
GAPDH 1457F & GAAGGTGAAGGTCGGAGTC & $1457-1475$ \\
GAPDH 3412R & GAAGATGGTGATGGGATTTC & $3412-3392$ \\
GAPDH probe & CAAGCTTCCCGTTCTCAGCC & $3363-3382$ \\
\hline
\end{tabular}


for $1 \mathrm{~min}$. The primers and probes for real-time RT -PCR for DSPP, DSP, ALP, and DMP-1 were designed using Oligo version 4.0 (National Bioscience, Plymouth, MN, USA) according to Heid et al.(18). A reporter oligonucleotide probe labeled at the 5', -end with a fluorescent reporter dye and at the 3' -end with a fluorescence quencher dye was designed to bind to the product amplified by the oligonucleotide primer pair. The sequences of all oligonucleotides used are shown in Table 1. The GAPDH primer and probe (TaqMan GAPDH detection reagents) were purchased from Applied Biosystems (Applied biosystems, Foster, USA). Signals were analyzed with the sequence detector 1.0 program (Perkin-Elmer). The amount of each mRNA was determined by comparing the real-time RT-PCR signals to a standard curve of signal vs. human bone marrow t-RNA (Clontech, Palo Alto, CA, USA) at different concentrations (4000 ng, $2000 \mathrm{ng}, 1000 \mathrm{ng}$, $500 \mathrm{ng}, 250 \mathrm{ng}, 125 \mathrm{ng}$, and $62.5 \mathrm{ng}$ ). The expression coefficients for each sample were determined by dividing the amount of specific mRNA by the amount of GAPDH mRNA.

\section{Results}

Morphology of DFCs, DPSCs, and OMCs in culture

We first observed the morphology of DFCs, DPSCs, and OMCs by phase contrast microscopy (Figs. 2A-C). These cells exhibited like spindleshaped and fibroblastic form in IM. More bone nodules were found in DFCs than in DPSCs, and none were found in OMCs in IM.

Odontoblast-specific and osteogenesis-related gene expression in DFCs, DPSCs, and OMCs in culture

To investigate the ability of DFCs, DPSCs, and OMCs to form dentin, we examined the expression of odontoblast-specific (DSPP and DSP) and osteogenesis-related (ALP, DMP-1) genes using real time RT -PCR. Steady-state expression of GAPDH mRNA was used as a control for expression, and similar levels of expression were found throughout the different experimental conditions.

Expression of the DSPP gene was observed in
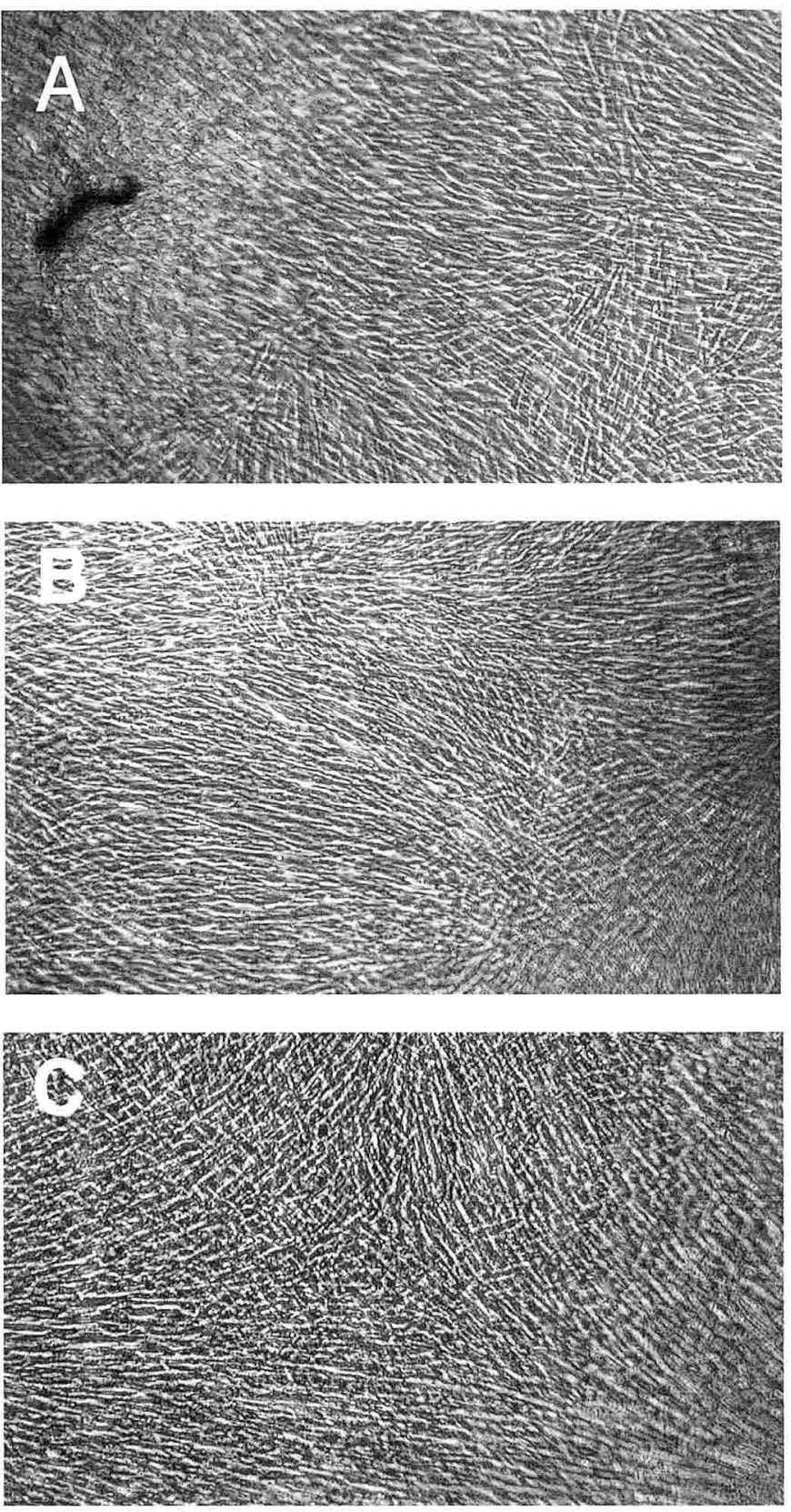

Fig. 2. Phase-contrast images of DFCs, DPSCs, and OMCs after 15 days in IM. DFCs: bone nodules are often found (A). DPSCs : appear as fibroblast-like cells (B). OMCs : bone nodules are not found (C). In all panels, magnification : $20 \mathrm{X}$.

DFCs at later times (day 6) of growth in IM. The coefficient of expression in DFCs and DPSCs increased with time, but the expression of DSPP in OMCs was nearly undetectable. After 3, 6, 9, 15, 21, and 27 days in IM, the expression coefficients in DFCs were $0.28,0.75,1.08,1.52,2.85$, and 5.80 , respectively. Thus, the value at day 27 was 20.7 -fold higher 
DSPP

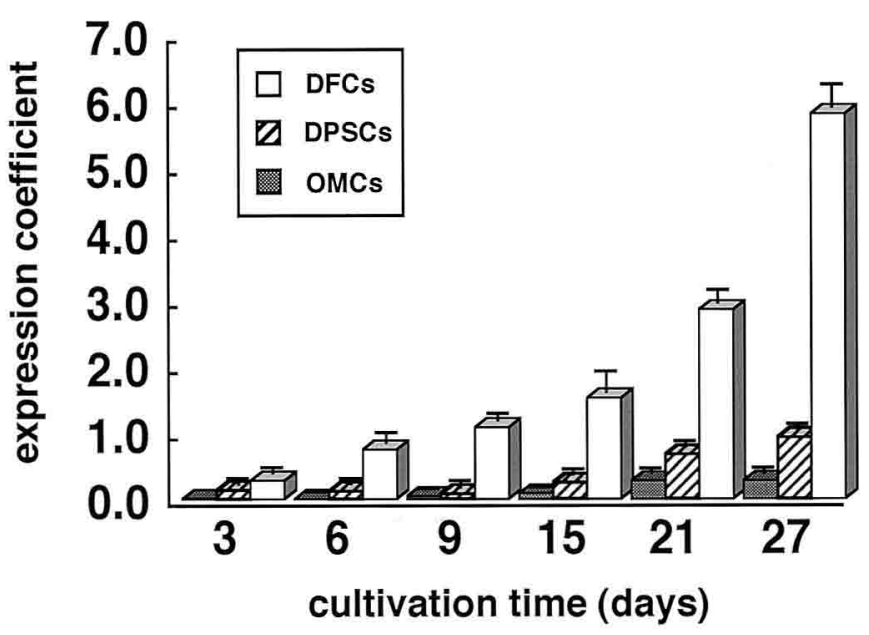

Fig. 3. DSPP gene expression in DFCs (open squares), DPSCs (striped squares), and OMCs (closed squares). After 3, 6, 9, 15, 21 , and 27 days, cells were lysed, and total RNA was extracted. Equal amounts of total RNA (200 ng) were analyzed by real -time RT-PCR, and the amounts of mRNAs for DSPP and GAPDH in each sample were determined by comparing the signals to standard curves. The expression coefficient for mRNA on the ordinate was calculated for each sample by dividing the amount of DSPP mRNA by the amount of GAPDH mRNA. Each point is the mean value obtained from three independent experiments. And the differences were less than $10 \%$ in the three experiments. Each bar represents the mean, and the error bars represent the mean \pm SD.

than the value at day 3. In DPSCs, we found expression of this gene after 15 days in IM, but the increase in expression was much slower. After 15 days, the expression coefficient in DFCs was also 6.17-fold higher than in DPSCs, and after 27 days, the expression coefficient was 6.26-fold higher in DFCs than in DPSCs. Finally, there was almost no expression of this gene in DFCs, DPSCs, or OMCs grown in CM (Fig. 3).

Expression of the DSP gene was observed in both DFCs and DPSCs grown in IM, with a slow increase until day 15. In DFCs, after 21 days in IM, there was a rapid increase in DSP gene expression. In DFCs, after $3,6,9,15,21$, and 27 days in IM, the expression coefficients were $0.33,0.39,0.60,0.86,3.55$, and 4.79 , respectively. Therefore, the value after 27 days was 14.5-fold higher than the value after 3 days. In addition, the expression coefficient for DSP in DFCs was 4.55-fold higher than the value in DPSCs after 21, and 4.00-fold higher than after 27 days. Almost no

\section{DSP}

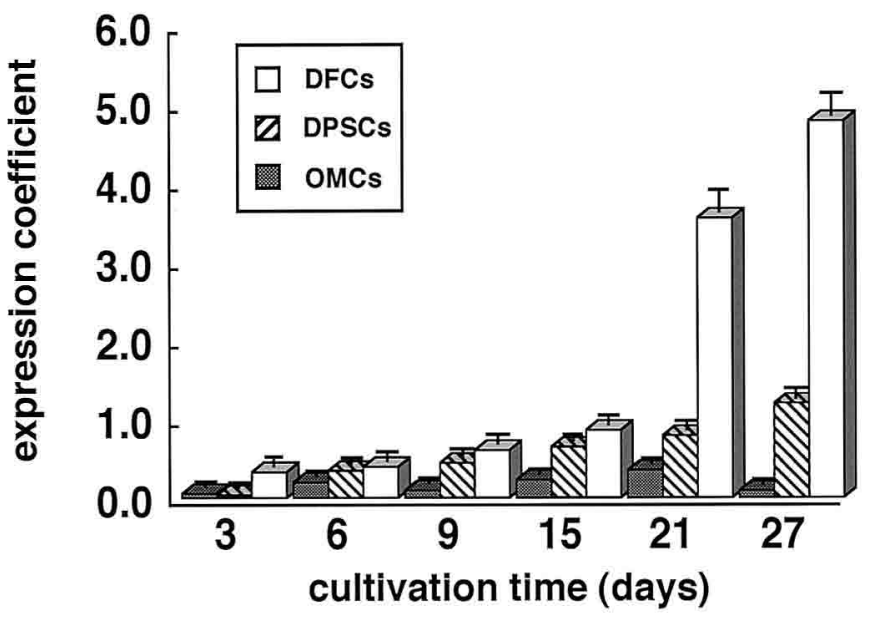

Fig. 4. DSP gene expression in DFCs (open squares), DPSCs (striped squares), and OMCs (closed squares). Experiments were performed and analyzed as in Fig. 3 except that DSP and GAPDH gene expression were measured.

expression of the DSP gene was found in OMCs in IM or in any of the three cell types in CM (Fig. 4).

Until 15 days of growth in IM, there was almost no expression of the ALP in the DPSCs and DFCs, but after 21 days, there was a time-dependent increase in the expression coefficient in DPSCs and DFCs. The expression coefficients were 0.49 and 1.15 and after 21 days and 0.98 and 4.37 after 27 days in DPSCs and DFCs, respectively. Therefore, after 21 days, the expression coefficient for ALP in DFCs was 2.35-fold higher than in DPSCs, and, after 27 days, 4.46-fold higher in DFCs than in DPSCs. Almost no ALP gene expression was observed in OMCs in IM or in any of the cell types in CM (Fig. 5).

Expression of the DMP-1 gene in DFCs and DPCs grown in IM appeared after 21 days and increased with time thereafter. The expression coefficients for DMP-1 gene expression were 0.70 and 1.29 after 21 days and 0.90 and 3.49 at 27 days in DPSCs and DFCs, respectively. Thus, the expression coefficient in DFCs after 21 days in IM was 1.84-fold higher than in DPSCs, and after 27 days, the expression coefficient was 3.88 -fold higher than in DPSCs. There was almost no DMP-1 gene expression in OMCs grown in IM or in any of the three cells when grown in $\mathrm{CM}$ (Fig. 6). 


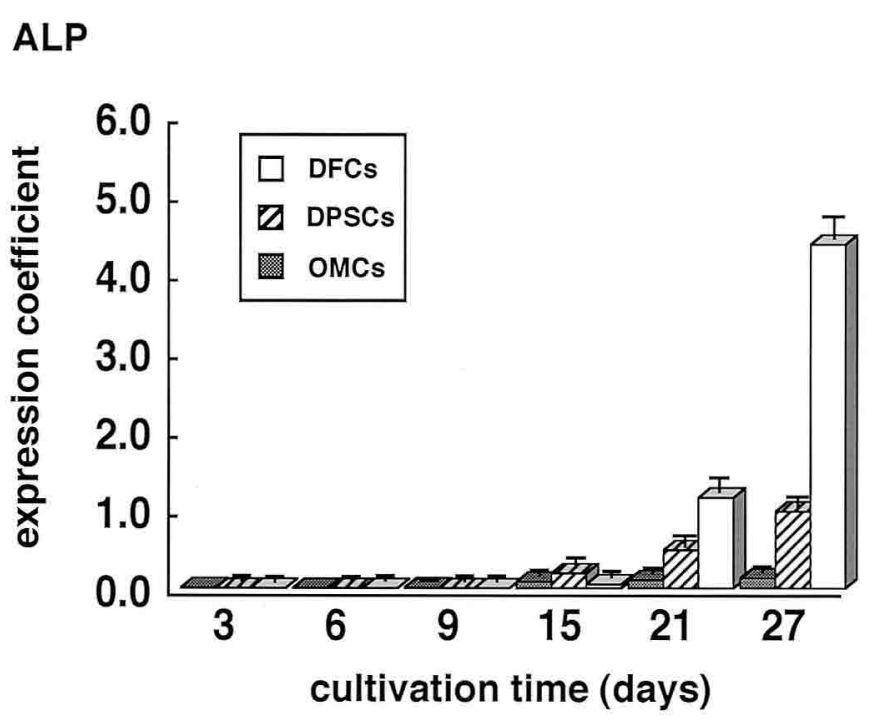

Fig. 5. ALP gene expression in DFCs (open squares), DPSCs (striped squares), and OMCs (closed squares). Experiments were performed and analyzed as in Fig. 3 except that ALP and GAPDH gene expression were measured.

\section{DMP-1}

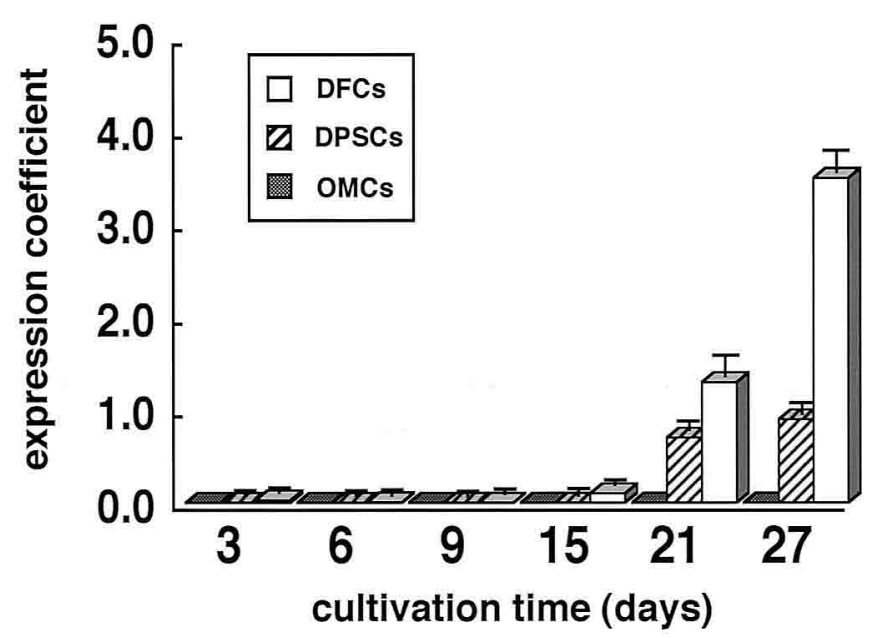

Fig. 6. DMP-1 gene expression in DFCs (open squares), DPSCs (striped squares) and OMCs (closed squares). Experiments were performed and analyzed as in Fig. 3 except that DMP-1 and GAPDH gene expression were measured.

Overall, these results show that DSPP, DSP, ALP, and DMP-1 genes were expressed in DFCs and DPSCs at later times when they were grown in IM. Expression of these genes was nearly undetectable in OMCs grown in IM, and in all three cell types grown in $\mathrm{CM}$.

\section{Discussion}

In this study, we have examined the ability of DFCs, DPSCs, and OMCs to form dentin by following their expression of four dentin and bone extracellular matrix genes, DSPP, DSP, ALP and DMP-1. Our results show that these genes are expressed at later times when DFCs and DPSCs are cultured in IM, but are not expressed when DFCs and DPSCs are cultured in CM, and with different patterns. In phase contrast microscopy, bone nodules were found in DFCs and DPSCs, and none were found in OMCs in IM cultivation. This result might indicate that DFCs and DPSCs have the ability of forming the mineralized tissues.

High levels of DSPP expression have been shown to persist in functional odontoblasts throughout all phases of primary dentinogenesis and in secondary dentinogenesis (17). Also, the expression of DSPP mRNA has been shown to be tooth-specific, to remain high in odontoblasts during and after completion of primary dentinogenesis, and to be more fundamentally involved than DMP-1 in the formation and maintenance of dentin. DSPP transcripts have also been detected in polarizing odontoblasts and in functional odontoblasts depositing the extracellular matrix components of predentin-dentin (20). Furthermore, DSPP and DMP-1 participate in a regulatory pathway required for the growth and differentiation of odontoblasts (21) and play a role in the mineralization of dentin (22).

In the current study, we showed that DSPP gene expression in DFCs and DPSCs occurs at early time points in IM (6 days), whereas DMP-1 and ALP gene expression appeared after 15 days. We also showed that expression of these genes in DFCs and DPSCs increased with time. Therefore, based on DSPP and DMP-1 expression, DFCs and DPSCs may correspond with the mineralization of odontoblasts. We further showed that the expression coefficient of DSPP in DFCs was 6.17-fold higher than in DPSCs after 15 days in IM, and 6.26-fold higher after 27 days. This suggests that, compared to DPSCs, DFCs have a superior ability to form dentin and contain more pre-odontoblast cells. 
Recently, in situ hybridization studies have demonstrated that DMP-1 is expressed in odontoblasts, ameloblasts, cementoblasts, and osteoblasts. It was also noted that DMP-1 is expressed not only in dentin but also on other mineralizing tissues (23). In the current study, we found that DMP-1 expression in DFCs and DPSCs increases at later times in IM. Therefore, DFCs and DPSCs may have the ability to mineralize at these later times. This is also supported by the expression of ALP in DPSCs and DFCs after 21 days in IM because this gene is an early maker for both osteoblasts and odontoblasts, plays a vital role in calcified tissue formation, and is thought to induce odontoblast differentiation (24). These results suggest that DFC and DPSC cultures contain cells that can differentiate into osteoblasts and odontoblasts after long times of continuous culture in IM. Finally, the patterns of DMP-1 and ALP gene expression were very similar, suggesting that their expressions are somehow related.

There was nearly no expression of the DSPP, DSP, ALP, or DMP-1 genes in OMCs or in DFCs or DPSCs grown in IM. This suggests that the cultivated cells might be differentiated into osteoblastic or odontoblast-like cells by the IM. This implies that the three supplements (dexamethasone, $\beta$-glycerophospate, and ascorbic acid-2-phosphate) must be added to the CM to give the DFCs or DPSCs the ability to form dentin or bone. And there may be no stem cells in OMCs which was stimulated to form mineralized tissue by IM.

In mice, the expression of DSP in dental pulp appears after odontoblast terminal differentiation and concurs with pre-dentin formation before the onset of mineralization (14). In situ hybridization in adjacent tissue sections show that DSP is deposited when dentin components are secreted and are progressively converted into dentin. However, the function of DSP remains unclear. In the current study, DSP gene expression in DFCs was observed after 21 days of growth in IM, and the gene expression pattern was similar to that of ALP and DMP-1 but different than DSPP. Based on these findings, we speculate that the DSP gene is induced at the initial phase of and participates in dentin mineralization.

In conclusion, our data demonstrate that DFCs should have the ability to differentiate into odontoblasts and that they contain more pre-odontoblast cells than DPSCs. Based on their expression of the DSPP, DSP, and DMP-1 genes, DFCs and DPSCs may have the ability to form dentin. In the future, DFCs may be useful in the engineering of dental materials and in the preservation and regeneration of dentin and teeth.

\section{Acknowledgments}

We are grateful to Dr. Hideharu Hibi, at the Center for Genetic and Regenerative Medicine, Nagoya University Hospital, and we thank the members of the Department of Oral and Maxillofacial Surgery at the Nagoya University Graduate School of Medicine. This work was party supported by a Grant-in-Aid for young scientists (B) (15791163) from the Ministry of Education, Culture, Sports, Science and Technology (MEXT) and by a Grant-in-Aid for Scientific Research (B) (16390583) from the Japan Society for the Promotion of Science (JSPS).

\section{References}

1. Reddi A : Role of morphogenic proteins in skeletal tissue engineering and regeneration. Nature Biotechnol, 16: 247-252, 1998.

2. Yamada Y, Ueda M, Naiki T, Takahashi M, Hata K, Nagasaka T : Autogenous injectable bone for regeneration with mesenchymal stem cells (MSCs) and platelet-rich plasma (PRP)-Tissue-engineered bone regeneration. Tissue Eng, 10: 955-964, 2004.

3. Yamada Y, Ueda M, Naiki T, Nagasaka T: Tissue ${ }^{-}$ engineered injectable bone regeneration for osseointegrated dental implants. Clin Oral Impl Res, in press (2004).

4. Yamada Y, Ueda M, Hibi H, Nagasaka T: Translational research for injectable tissue-engineered bone regeneration using mesenchymal stem cells and platelet-rich plasma from basic research to clinical case study. Cell Transplant, in press.

5. Ueda M, Yamada Y, Ozawa R, Okazaki Y : A clinical report of injectable tissue-engineered bone applied for alveolar augmentation with simultaneous implant placement. Int J Periodontics Resrtative Dent, in press.

6. Ozawa R, Yamada Y, Nagasaka T, Ueda M : A com- 
parison of osteogenesis-related gene expression of mesenchymal stem cells during the osteoblastic differentiation induced Type-I collagen and/or fibronectin. Int J Oral-Med Sci, 1: 148-155, 2003.

7. Yamada $Y$, Itano N, Hata K, Ueda M, Kimata K : Differential regulation by IL- $1 \beta$ and EGF of expression of three different hyaluronan synthases in oral mucosal epithelial cells and fibroblasts and dermal fibroblasts : quantitative analysis using real time RTPCR. J Invest Dermatol, 122 : 631-639, 2004.

8. Ueda M, Hata K, Horie K, Torii S: The potential of oral mucosal cells for cultured epithelium: A preliminary report. Ann Plast Surg, 35 : 498-504, 1995.

9. Tziafas D, Smith AJ, Lesort H : Designing new treatment strategies in vital pulp therapy. J Dent, 28: 7792, 2000 .

10. Gronthos S, Mankani M, Brahim J, Robey PG, Shi S : Postnatal human dental pulp stem cells (DPSCs) in vivo and in vitro. Proc Natl Acad Sci USA, 97 : 13625 -13630, 2000.

11. Wise G, Hung H, Que B : gene expression of posternal tooth eruption molecules in the dental follicle of the mouse. Eur J Oral Sci, 107 : 482-486, 1999.

12. Hanada K, Saito M, Yamauchi M, Kiyono R, Sato S, Taranaka T, Narayanan A: Cementum matrix formation in vivo by cultured dental follicle cells. Bone, 31: 606-611, 2002.

13. Gluhak HJ, Ye L, Onewald LF, Jian QF, MacDougall M, Harris S, Pavlin D: Mechanical loading stimulates Dentin Matrix Protein a (DMP 1) expression in osteocytes in vivo. J Bone Miner Res, 18: 807-813, 2003.

14. D'Souza RN, Bronckers ALJJ, Happonen RP, Doga DA, Farach-Carson MC, Butler WT: Deveropmental expression of $53 \mathrm{kDa}$ dentin sialoprotein in rat tooth organs. J Histochem. Cytochem, 40 : 359-366, 1992.

15. Stein GS, Lian JB, Owen TA : Relationship of growth to the regulation of tissue specific gene expression during osteoblast differentiation. FASEB J, 4: 31113123, 1990.

16. George A, Sabsay B, Simonian PAL, Veis A : Charac- terization of a novel dentin matrix acidic phosphoprotein. Implications for induction of biomineralization. J Biol Chem, 268 : 12624-12630, 1993.

17. D'Souza R, N Cavender A, Sunavala G, Alvarez J, Ohshima T, Kukakarni A, Macdougall M: Gene expression patterns of murine dentin matrix protein 1 (DMP-1) and dentin sialophosphoprotein (DSPP) suggest distinct development functions in vivo. J Bone Miner Res, 12 : 2040-2049, 1997.

18. Heid CA, Stevens J, Livak KJ, Wulliums OM : Real time quantitative PCR. Genome Res, 6 : 986-994, 1996.

19. Gibson UEM, Heid CA, Willoams A: Novel method for real time quantitative RT-PCR. Genome Res, 6 : 995-1001, 1996.

20. Begue K, Krebsbach PH, Bartlett JD, Bulter WT: Dentin sialoprotein, dentin phosphoprotein, enamelysin and ameloblastin: tooth-specific molecules that are distinctively expressed during murine dentin differentiation. Eur J Oral Sci, 106 : 963-970, 1998.

21. Karthikeyan N, Rampalli S, Amsaveni R, Jianjun H, Bruce Q, Anne G : Differentiation of embryonic mesenchymal cells to odontoblast-like cells by overexpressions of dentin matrix protein. Proc Natl Acad Sci USA, 98: 4516-4521, 2001.

22. D'souza RN, Cavender A, Sunavala G, Alvarez J, Ohshima T, Kulkarni AB Macdougal M: Gene expression patterns of murine dentin matrix protein 1 and dentinsialophospho protein suggest distinct development functions in vivo. J Bone Miner Res, 12: 2040 $-2049,1997$.

23. Macdougall M, Gu TT, Luan X, Simmons D, Chen J : Identification of novel isoform of mouse dentin matrix protein 1: special expression in mineralized tissues. J Bone Miner Res, 13 : 422-431, 1998.

24. Owen TA, Aronow M, Shalhoub V, Strein GS : Progressive development of the rat osteoblast phenotype in vitro : reciprocal relationship in expression of genes associated with osteoblast proliferation and differentiation during formation of the bone extracellular matrix. J Cell Physiol, 143 : 420-430, 1990. 